\title{
Inverse Modeling Using Taylor Expansion Approach and Jacobi Matrix on Magnetic Data (Dike/Magma Intrusion Cases)
}

\author{
Agus Suprianto $^{1, a}$, Nurul Priyantari ${ }^{1, b}$, Supriyadi $^{1, \mathrm{c}}$, Aryono Adhi ${ }^{\text {2,d, }}$ \\ Wahyudi $^{3, e}$, Wiwit Suryanto ${ }^{3, f}$, Ari Setiawan ${ }^{3, g}$, and Agus Subekti ${ }^{1, h}$ \\ ${ }^{1}$ Physics Dept, Faculty of Mathematics and Natural Sciences, Universitas Jember, Indonesia \\ ${ }^{2}$ Physics Dept, Faculty of Mathematics and Natural Sciences, Universitas Negeri Semarang, Indonesia \\ ${ }^{3}$ Physics Dept, Faculty of Mathematics and Natural Sciences, Universitas Gadjah Mada, Indonesia

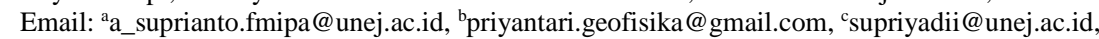

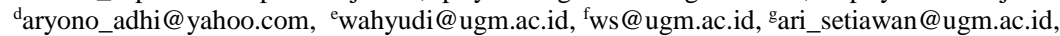 \\ hsubekti@unej.ac.id
}

\begin{abstract}
The mathematical modeling of dike or magma intrusion has been done, based on magnetic data with inversion techniques using MatLab. The magnetic equation was done using a linear approach to non-linear mathematical models of magnetic data using the Taylor expansion approach and Jacobi Matrix. The first step, we made a synthetic data forward modeling from the magnetic equation of dike or magma intrusion cases without errors, and then add errors to the data. The next step was to do an inversion to get the parameters sought, i.e., depth and angle of the magma intrusion, by giving initial guesses, and then recorrect solution iteratively until obtained the convergent results. Finally, parameters of dike/magma intrusion slope orientation and its depth can be determined, and this technique can be used to get the physical parameters of these geological cases.
\end{abstract}

Keywords: Inverse modeling, Taylor expansion, Jacoby matrix, magnetic data, dike, magma intrusion.

\section{INTRODUCTION}

The objective of measuring using geophysical methods is to get a subsurface model of observational field data, i.e., magnetic susceptibility, density, resistivity, and others [1]. A magnetic method is one of the geophysical methods based on measuring the variation of magnetic field intensity on the surface of the earth caused by differences in the distribution of magnetized sub-surface objects [2], using magnetic sensors, in order to determine the rocks magnetic susceptibility [3]. This difference in susceptibility can occur when a rock is broken through by other rocks as well as a magmatic intrusion of maar and cinder cone [4] as well as at Mount Lamongan area [5]. A mathematical formulation is needed to connect the sub-surface susceptibility model and observation data at the surface [6], using forward and inversion modeling [7]. Forward modeling is done by calculating the anomaly of the model and comparing it with the anomaly data from the measurement results, while inversion modeling calculates the value of physical parameters from the existing observation data based on the mathematical model. The main difficulty occurs when we do inversion modeling at non-linear 
mathematical equations such as mathematical magmatic intrusion equations so that the solution no longer uses a linear approach [8]. Some solutions to this problem can be done using the simulated annealing approach [9] and TaylorJacobi expansion [7]. In this paper, we use Taylor-Jacobi expansion iteratively to solve non-linear equations of dike/magmatic intrusion formula in order to determine its parameters, i.e., slope orientation and depth.

\section{METHODS}

The mathematical equations formulated by [10] were then modified by [11], and Almantouq [12] used to get synthetic data on geological structures, i.e., dike or magma intrusion. From this synthetic data, a subsurface structure model, i.e., orientation and depth, can be determined using the inversion method with Taylor Expansion Approach and Jacoby Matrix.

\subsection{Anomaly magnetic field formulation due to dike/magmatic intrusion}

Dike and magma intrusion is a sheet of rock that fills or breaks through existing rock bodies [12]. Frozen rocks shaped flat in the form of these plates occur because of the process of intrusion of magma between existing rock layers either with upright or tilted directions. [10] in [8] explain the relationship of magnetic anomaly field $F(x)$ caused by an infinite dike with a certain depth, can be written as,

$$
\begin{aligned}
& F(x)=C\left[\cos \alpha\left\{\tan ^{-1}\left(\frac{x+b}{h}\right)-\tan ^{-1}\left(\frac{x-b}{h}\right)\right\}+\right. \\
& \left.\cdots \frac{1}{2} \sin \alpha \ln \left\{\frac{(x+b)^{2}+h^{2}}{(x-b)^{2}+h^{2}}\right\}\right]
\end{aligned}
$$

$F(x)$ is a magnetic field profile measured along $\mathrm{x}$, and perpendicular to the strike direction of the magma intrusion. For charts with tiny widths and depths, equation (1) above written as [10,11],

$$
F(x)=C\left[\frac{x \sin \alpha+h \cos \alpha}{x^{2}+h^{2}}\right]
$$

With $\alpha$ is the angle formed by horizontal to dike or thin magma column, $\mathrm{x}$ is the position of the measurement offset data, $\mathrm{h}$ is the depth of the dike, and $\mathrm{C}$ is the constant (3225 nT). Using equation (2) above, we can calculate the magnetic field caused by forward modeling along with the offset of $\mathrm{x}$ measurement.

\subsection{Mathematical Equations of Inversion Method}

In general, inversion techniques are formulated in the following relationships $[7,14]$,

$$
d=G(m)
$$


with $d=\left[d_{1}, d_{2}, d_{3}, \ldots, d_{N}\right], G$ is a general function and $m=\left[m_{1}, m_{2}, m_{3}, \ldots\right.$, $\left.m_{N}\right]$ is a model parameter. By multiplying the two segments $d=G m$ (equation 3) with $G^{T}$ then we get,

$$
G^{T} d=G^{T} G m
$$

The above equation then we multiply the two sections again with $\left(G^{T} G\right)$ and we get,

$$
m=\left(G^{T} G\right)^{-1} G^{T} d
$$

The Equation (5) is an inversion for linear cases, while Equation (2) used for magnetic data forward modeling geological case studies are a case of non-linear equations. The kernel matrix, which is a function of forward modeling, no longer contains the predicted model parameters, which is then carried out by the process of matching the model parameters. Suppose the solution of inversion in Equation (3) is m which is the initial model $m_{0}$ which matched with $\Delta m$ in order to obtain a better match of the response of the model to the data [7], then

$$
m=m_{0}+\Delta m
$$

and from equation (3), by applying equation (6) for this case can be written as,

$$
d=G\left(m_{0}+\Delta m\right)
$$

and if the equation (15) rewritten at its components,

$$
d_{i}=G_{i}\left(m_{0}^{(j)}+\delta m_{j}\right)
$$

with $i=1,2,3 \ldots \mathrm{N}$ is the amount of data, and $j=1,2,3, \ldots, m$ is the model parameter.

The first-order Taylor Expansion of $G(\mathrm{~m})$ function around the model from equation (16) and ignore the residual tribe, then produce, [6],

$$
d_{i}=G_{i}\left(m_{0}^{(j)}+\left.\frac{\partial G_{i}}{\partial m_{j}}\right|_{m_{0}} \delta m_{j}\right)
$$


with $\frac{\partial G_{i}}{\partial m_{j}}$ is the Jacobi mabtrix,

$$
G_{i j}=\frac{\partial G_{i}}{\partial m_{j}}
$$

by substituting an equation (10) into equation (9) and rearranging equation (9) again, it will obtain,

$$
d_{i}-G\left(m_{0}^{(j)}\right)=G_{i j} \delta m_{j}
$$

So, the equation (19) can be written in matrix notation as follow,

$$
d-G\left(m_{0}\right)=G_{0} \Delta m_{0}
$$

or,

$$
\Delta d_{0}=G_{0} \Delta m_{0}
$$

or,

$$
\Delta d_{0}=d-G\left(m_{0}\right)
$$

Furthermore, $G_{0}$ is the Jacobi matrix which evaluated at $m=m_{0}$. The equation (13) is similar to $d=G(m)$, so by analogy with the same formula differentiation in equations (10) to (13), by multiplying the two segments of $\Delta d_{0}=G_{0} \Delta m_{0}$ (equation 21) with $G_{0}^{T}$, then we get,

$$
G_{0}^{T} \Delta d_{0}=G_{0}^{T} G_{0} \Delta m_{0}
$$

by multiplying two segments in equation (15) with $\left(G_{0}^{T} G_{0}\right)^{-1}$, then obtained,

$$
\left(G_{0}^{T} G_{0}\right)^{-1} G_{0}^{T} \Delta d_{0}=\left(G_{0}^{T} G_{0}\right)^{-1}\left(G_{0}^{T} G_{0}\right) \Delta m_{0}
$$

the matrix $\left(G_{0}^{T} G_{0}\right)^{-1}\left(G_{0}^{T} G_{0}\right)$ in equation (16) is an identity matrix, so that it equation can be rewritten as,

$$
\Delta m_{0}=\left(G_{0}^{T} G_{0}\right)^{-1} G_{0}^{T} \Delta d_{0}
$$

then, by substituting the equation (15) to (17), it is obtained, 


$$
\Delta m_{0}=\left(G_{0}^{T} G_{0}\right)^{-1} G_{0}^{T}\left(d-G\left(m_{0}\right)\right)
$$

By doing iteratively matching equation (25) to the initial model $m_{0}$ so that a better model obtained $\left(m=m_{0}+\Delta m_{0}\right)$. The model response is expected to be more appropriate and fit with subsurface geological features. The $G$ which is the Jacobi matrix in the magmatic intrusion case (equation 2) consists of,

$$
G=\left[G_{\alpha} G_{h}\right]
$$

with $G_{n}^{T}$ is a transpose matrix for each parameter in the $n$ iteration, $d$ is data and $G\left(m_{0}\right)$ is the inverse calculation model result. Jacobi matrix $G_{l}$ and $G_{2}$ expressed as equation (20) and (21).

$$
\begin{aligned}
& G_{1}=\frac{d F(x)}{d \theta}=\frac{-A \cdot((H \cdot \sin \theta-X \cdot \cos \theta))}{X^{2}+H^{2}} \\
& G_{2}=\frac{d F(x)}{d H}=\left(-A \cdot \frac{\cos \theta}{H^{2}+X^{2}}\right)-(2 A H \cdot H \cos \theta+X \cdot \sin \theta) /\left[X^{2}+H^{2}\right]^{2}
\end{aligned}
$$

\subsection{Magnetic data inversion step}

The first step is to make synthetic data $\left(d^{o b s}\right)$ at field data by carrying out a forward technique using equation (2) by using specific $\alpha$ and h model parameters. The data from the forward modeling then added to a random error. The second step is to make the same data as the first step (data $d^{\text {cal }}$ ) by making an initial guess at the model parameters $\alpha$ and $h$. The next step calculates the difference between $d^{\text {cal }}$ dan $d^{\text {obs }}$. If there is a difference (within specified limits), modifications are made to the stages of the model parameter values until appropriate. The step is shown by the flow chart of the image (1). When the difference between the actual parameters and the inversion results is very small and the inversion result parameters are correct, the $\alpha$ (dike/magma intrusion angle) and $\mathrm{h}$ (dike/magma intrusion depth) parameters obtained from the inversion process will be obtain.

\section{RESULT AND DISCUSSION}

After the algorithm and script program completed, the test is carried out with several stages; the first step is to create synthetic data from equation (2) in forward modeling. And verify the pattern of the forward model made with existing software that, in this case, we use Mag2DC to prove it. The next step is to create a forward model that added to a random error that seems to represent a field data. After that, we do an inversion script test by inversion the forward data that has not been given an error, assuming that if the program script created correctly, the result will be the same as the data forward. The last step is to test by inverting the forward data that has given a random error. 


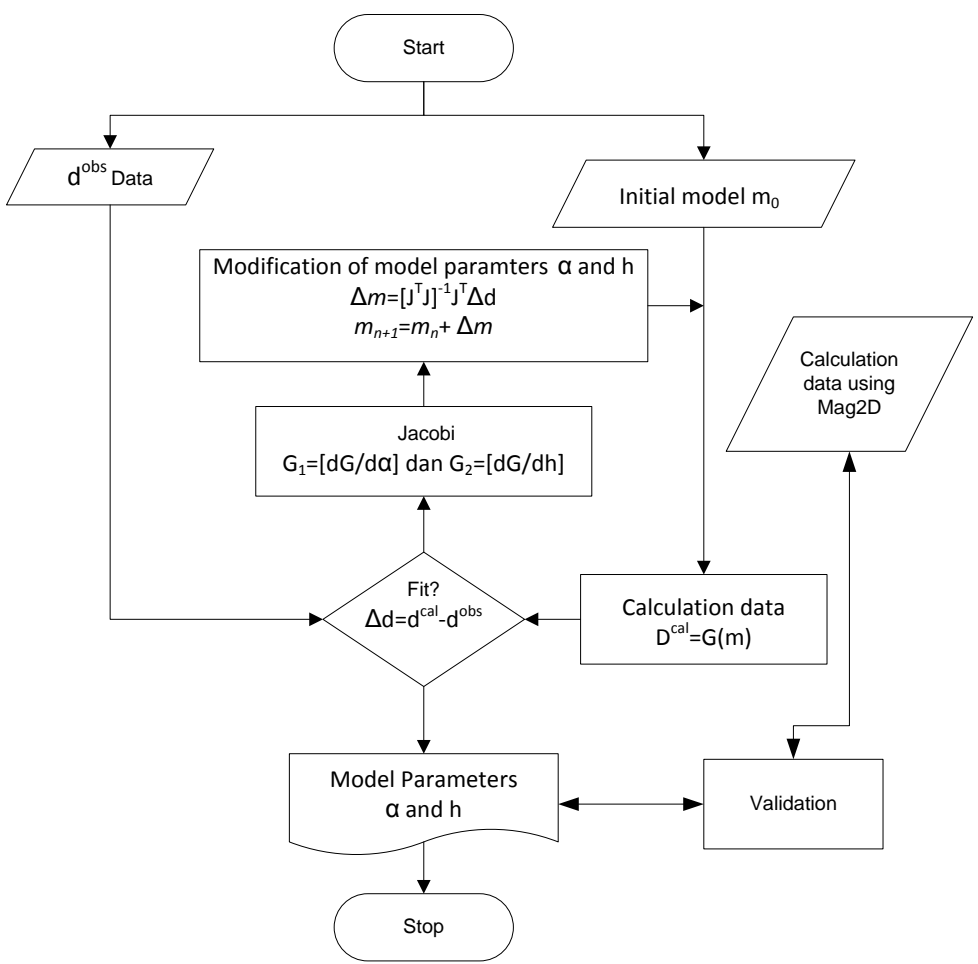

Figure 1. Flowchart of magnetic data inversion research

\subsection{Forward Modeling and verification}

After the algorithm and script program completed, several stages of the test were carried out. The first step is to create synthetic data from equation (2) in forward modeling to make a magnetic data of a simple dike case or thin magma intrusion/dike, verify the pattern of the forward model made with existing software that, in this case, we use Mag2DC to verify it. Mag2dc is software for modelling magnetic data and make a sub-surface model of magnetic data. First, the model made with $\alpha$ simple dike with an orientation of $60^{\circ}$ and $90^{\circ}$ at 160 meters depth, offset $\mathrm{x}$ from -5000 meters to 5000 meters with interval measuring points as far as 150 meters. Output, the results of the forward modeling are shown in figure (1) and (2). There is a suitability of the results of forward modeling with angular parameters of $60^{\circ}$ and $90^{\circ}$ at a depth of 160 meters (figures 1(a) and 2(a)), with the results of the forward model with the same parameters using the MAG2DC software (figures 1(a) and 2(b)). The forward yield graph pattern built using equation (2) produces results data known as synthetic observation.

\subsection{Forward Modeling added an error}


Because there is no direct field data/observation data, an error is added to the forward model data in the previous step as if it were field data or substitute for field data. The assumption used here is that field data is usually not ideal as a model formulation, but there is an error due to measurement. The forward result that has added to this error presented by a figure (3),

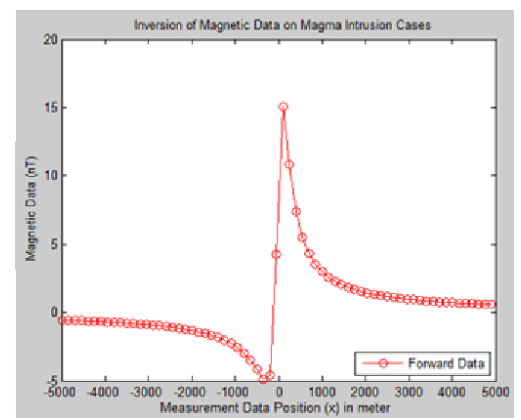

(a)

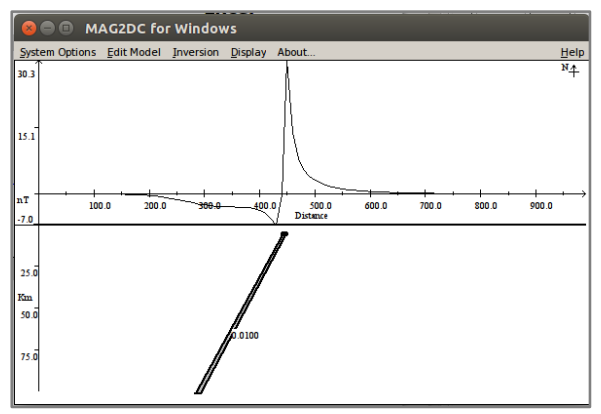

(b)

Figure 1. (a) Forward modeling with parameters $\alpha=60^{\circ}$ and h (dept of top dike or thin magma column) $=160$ meters, (b) Verify the forward model using Mag2DC at the same parameter of forward modeling $\left(\alpha=60^{\circ}\right.$ and $\mathrm{h}=160$ meters $)$.

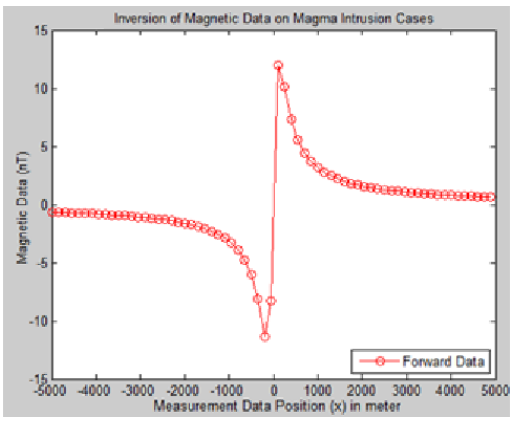

(a)

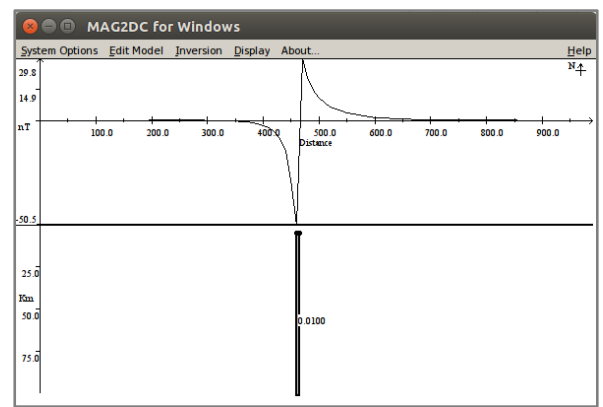

(b)

Figure 2. (a) Forward modeling with parameters $\alpha=90^{\circ}$ and $h=160$ meters, (b) Verify the forward model using Mag2DC with the same parameter of forward modeling $\left(\alpha=90^{\circ}\right.$ and $h=160$ meters $)$.

Data from the addition of errors is not smooth or not as ideal as the data before an error added. The model parameters used are at angle $\alpha=60^{\circ}$ and depth of dike (h) $=160$ meters. The same can done for several different parameters.

\subsection{Forward Data and Inversion Result without Error}

The next step is testing from the inversion process that has been made by making forward data and then making an inversion process. The inversion step will be correct if the final result of the model parameter inversion calculated is the same 
as the model parameter used during the forward process, and the data graph plot coincides.

In figure 4 is the result of a forward with an angle $\alpha=60^{\circ}$ and a depth of $\mathrm{h}=160$ meters. From the results of inversion with initial guessing approaching the forward value with $\alpha=65^{0}$ and $h=155$ meters, the inverse results are $\alpha=60,01$ and $h=160$ with a misfit of 0.000005 and 4 iteration processes. With the same parameters $\left(\alpha=60^{\circ}\right.$ and depth $\left.(h)=160\right)$ then inversion is done by using a rather distant guess that is $\alpha=80^{\circ}$ and depth is $\mathrm{h}=150$ meters. With this guess, the model parameters obtained from the inversion results are $\alpha=59.89$, and the depth is $h=159.90$ meters, with misfit $=0.73$ and need 7 iteration processes it. In the inversion process with different initial guessing parameters, the inversion results are almost the same but have differences in terms of misfit and time of the iteration process. In a distorted initial guess, the obtained misfit is more significant and requires a longer iteration time.

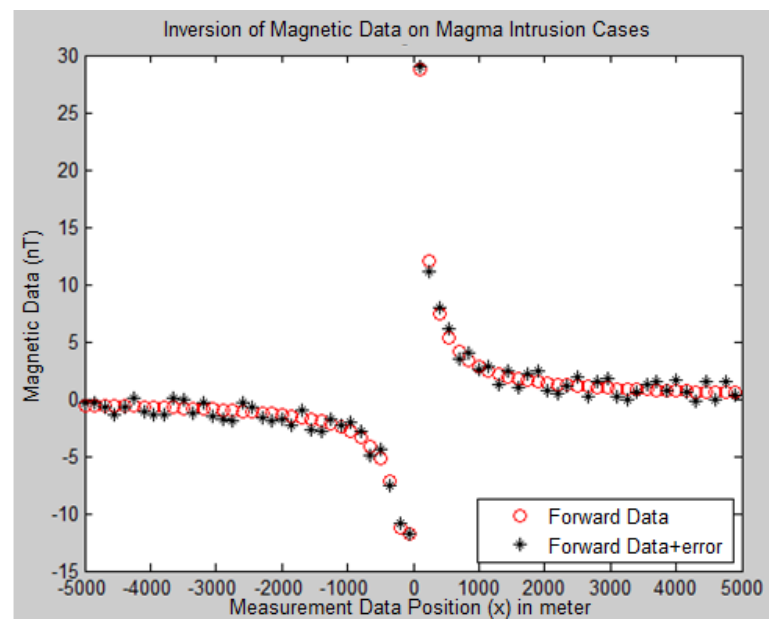

Figure 3. Forward data $\left(\alpha=60^{\circ}, \mathrm{h}=160 \mathrm{~m}\right)$ that has added an error. 


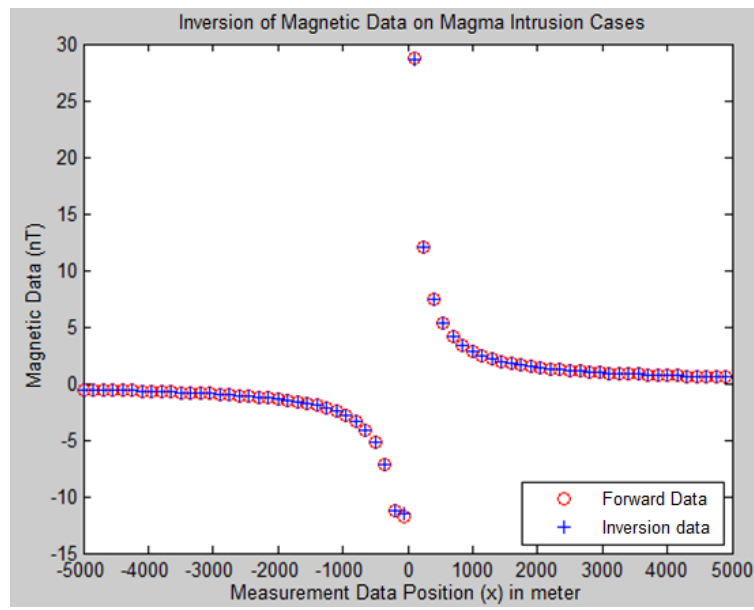

Figure 4. Forward, and Inversion result without errors $\left(\alpha=60^{\circ} ; \mathrm{h}=160 \mathrm{~m}\right)$.

At this stage, the inversion algorithm built can be used to guess the parameters of the slope angle and depth of the subsurface object with a simple dike or thin magma intrusion scenario in ideal conditions without the addition of errors in the data.

\subsection{Inversion Result with Error}

In this test, data forward is used, which has added an error in substitute for field data. At this stage, the first test is carried out by varying the different angles and fixed $h$, guessing with fixed angles and $h$ depth varies, and both are varied. In this first case example a trial with parameters $\alpha=100^{\circ}$ and $\mathrm{h}=300 \mathrm{~m}$, with an initial guess $\alpha=30^{\circ}$ and $\mathrm{h}=100 \mathrm{~m}$. The results of this inversion shown in figure 5 . The result of these processes shown that the model parameters obtained from inversion $\alpha=99.88 ; \mathrm{h}=299,57 \mathrm{~m}$, with misfit equal to 0.58 and the five iterations. This parameter occurs until the inversion model has a parameter similar to the forward parameter. The first iteration satisfaction is 4.40 , the second iteration $=3.40$, the third iteration $=2.38$, the fourth iteration $=1.40$ and the fifth iteration is obtained misfit $=0.58$. This misfit decays until the value of the misfit below one is obtained. 


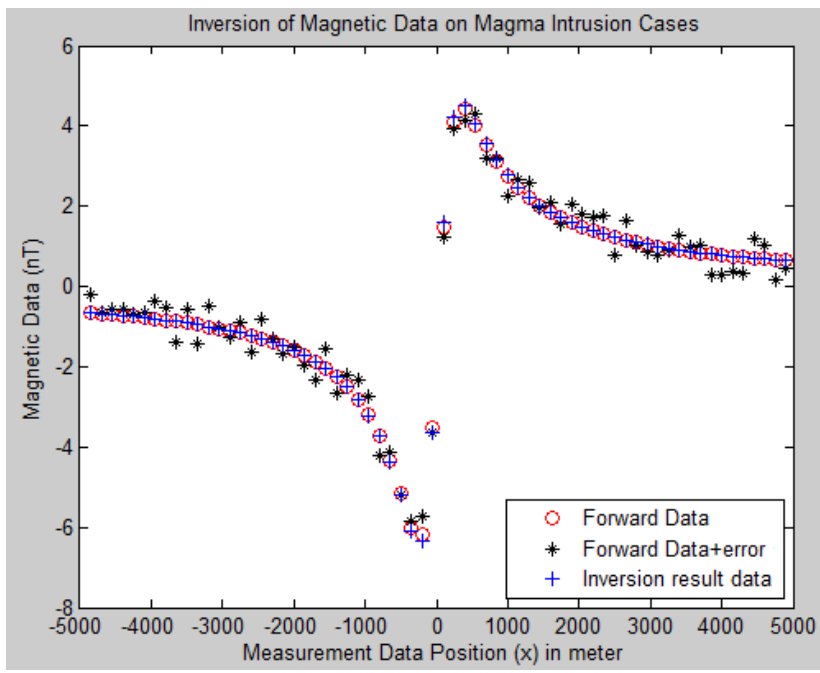

Figure 5. Forward and Inversion results with errors at $\alpha=100^{\circ}$ and $\mathrm{h}=300 \mathrm{~m}$, and initial guesses at $\alpha=30^{\circ} ; \mathrm{h}=100 \mathrm{~m}$.

\section{CONCLUSION}

The mathematical equation of the non-linear model can be solved using a linear approach with the Taylor expansion and the Jacobi Matrix iteratively. The inversion value at a far estimate of the model parameter will be valid if the initial guess is not too far away. At a far estimate of the initial parameter input, more iterations are needed to converge and need a more extended time to process it. Using this method, the simple sub-surface model of magmatic intrusion/dike parameters, i.e., orientation and depth, can be determined.

\section{REFERENCES}

[1] Milsom. J. (2003). Field Geophysics, Third Edition, John Wiley \& Sons LTD, Chichester, West Sussex, England.

[2] Hinze.W.J., Von Frese. R.R.B., \& Saad. A.H. (2013). Gravity and Magnetic Exploration, Cambridge University Press, New York.

[3] Reynolds. J.M., 1997, An Introduction to Applied and Environmental Geophysics, John Wiley \& Sons, New York.

[4] Blaikie.T.N., Ailleres.L., Betts.P.G., \& Cas. R.A.F. (2014). Interpreting subsurface volcanic structures using geologically constrained 3-D gravity inversion: Examples of maar-diatremes, Newer Volcanics Province, southeastern Australia.

[5] Suprianto.A., Wahudi, Suryanto.W., \& Subekti.A. (2015). Modeling of seismic wave propagation at Tiris Geothermal Area using 2D Spectral Element Method (SEM). Applied Mechanics and Materials, 771, 257-260.

[6] Vogel. C.R. (2002). Computational Methods for Inverse Problem, Society for Industrial and Applied Mathematics, Philadelphia. 
[7] Grandis. H. (2009). Pengantar Pemodelan Inversi, Himpunan Ahli Geofisika Indonesia (HAGI), Bhumi Printing, Bandung.

[8] Beiki. M., \& Pedersen. L.B. (2011). Estimating magnetic dike parameters using a non-linear constrained inversion technique: an example from the Sarna area, West Central Sweden, Geophysical Prospecting, European Association of Geoscientists \& Engineers (EAGE), September.

[9] Sharma. S.P., \& Kaikkonen. P. (1998) Two-dimensional non-linear inversion of VLF-R data using simulated annealing. Geophys. J. Int, 133, 649-668.

[10] Grant, F.S. \& West, G.F. (1965). Interpretation Theory in Applied Geophysics, McGraw-Hill Book Company, New York.

[11] Rambabu. H.V.,\& Sinha. G.D.J.S. (1986). Magnetic Anomalies Over Thin Plates and Their Analysis. Proc. Indian Acad. Sci. (Earth Planet. Sci), 95(3), 331-341.

[12] Dondurur.D., \& Oya Ankaya Pamukcu. (2003). Interpretation of Magnetic Anomalies from Dipping Dike Model Using Inverse Solution, Power Spectrum and Hilbert Transform Methods. Journal of the Balkan Geophysical Society, 6(2), 127-136.

[13] Wikipedia (without years) asessed from https://en.wikipedia.org/wiki/Dike_ (geology)

[14] Ricardson R.M. \& Zandt. G. (2009). Inverse Probles in Geophysics GEOS 567, Tucson, Arizona. 